\title{
Describing the Homeless Mentally Ill: Cluster Analysis Results ${ }^{1}$
}

\author{
Carol T. Mowbray ${ }^{2}$ \\ Wayne State University School of Social Work
}

\section{Deborah Bybee}

Michigan State University

Evan Cohen

The University of Michigan Medical Center

Presented descriptive data on a group of homeless, mentally ill individuals $(\mathrm{N}=108)$ served by a two-site demonstration project, funded by NIMH. Comparing results with those from other studies of this population produced some differences and some similarities. Cluster analysis techniques were applied to the data, producing a 4-group solution. Data validating the cluster solution are presented. It is suggested that the cluster results provide a more meaningful and useful method of understanding the descriptive data. Results suggest that while the population of individuals served as homeless and mentally ill is quite heterogeneous, many have well-developed functioning skills - only one cluster, making up $35.2 \%$ of the sample, fits the stereotype of the aggressive, psychotic individual with skill deficits in many areas. Further discussion is presented concerning the implications of the cluster analysis results for demonstrating contextual effects and thus better interpreting research results from other studies and assisting in future services planning.

\footnotetext{
${ }^{1}$ This research was supported in part by the National Institute of Mental Health Grant H87 MH44373, to the Michigan Department of Mental Health.

${ }^{2}$ All correspondence should be addressed to Carol T. Mowbray, Wayne State University, School of Social Work, 4756 Cass Avenue, Detroit, Michigan 48202.
} 
In the last decade, descriptive research studies on the homeless, particularly the homeless mentally ill, have proliferated. Some consensus has been reached concerning population characteristics. As summarized by Dennis, Buckner, Lipton, and Levine (1991), approximately one third of single adult homeless individuals have a serious mental illness. Studies seem to agree that, among the homeless, the mentally ill have been homeless longer and more frequently, have less contact with family and friends and fewer social supports and networks, are in poorer physical health, experience more frequent contacts with the criminal justice system, and are often treatment noncompliant and abusing of substances. In demographic characteristics, they present many similarities to the nonmentally ill homeless (e.g., usually under age 40 , predominantly male, disproportionately of minority status, and mainly a nontransient population).

However, even these "consensus" characteristics show some variation across studies. For example, Tessler and Dennis (1989), reviewing the first round of NIMH-funded studies, indicated that, for the homeless mentally ill, the proportion of nonwhite participants ranged from 33\% in a Milwaukee study to $79 \%$ in New York City research; and physical health problems ranged from 37 to $51 \%$ across studies. The percentage using alcohol and/or drugs ranged from 8 to $43 \%$. Fischer's (1989) comprehensive review of epidemiological studies of homelessness reports even wider ranges. Across all studies, alcohol problem prevalence ranged from 4 to $86 \%$; other drugs from 1 to $70 \%$. Restricting the review to only those studies of shelter populations reduced the range somewhat: alcohol, 7 to $86 \%$; other drugs, 1 to $61.2 \%$. Selecting only those studies of shelter users which employed standardized scales drastically reduced the number of studies but still produced wide ranging prevalence figures: eight alcohol studies, 28.3 to $66.8 \%$; two drug studies, 10.1 to $48.0 \%$.

Although methodological and instrumentation problems are significant obstacles to consistency in characterizing individuals who are homeless and mentally ill (Fischer \& Breakey, 1991), it seems likely that these dramatic variations reflect more than differences in research methods. Certainly, any researcher who has spent time working with the homeless from diverse referral sites (e.g., shelters, soup kitchens, psychiatric crisis centers) or various geographical locations will agree that these subgroups are different in substantive ways.

Descriptive research, at this point, however, seems "stuck" in being able to assist in meaningful interpretation of these differences across studies. For example, Sosin, Piliavin, and Westerfelt (1990), in studying two samples of homeless individuals in Minneapolis, note that it is "troubling that several characteristics of members in both samples seem to vary from those found in other studies" (p. 163). Some of the 
differences were explained in terms of the Minneapolis area population characteristics; others could not be (e.g., underrepresentation of women; overrepresentation of serious drinking problems). The field will not advance much in understanding the characteristics of homeless mentally ill individuals and possible contextual effects if we continue to generate research data that merely describe this population on a variety of measures, then compare a plethora of output to previous reports in the literature, and attempt to generate post-hoc explanations. We need more summative approaches to analysis and interpretation to improve our ability to interpret these differences and therefore utilize findings relevant to prevention and/or treatment.

A method is needed to organize and identify patterns within the vast array of information collected on individuals who are homeless and mentally ill. Data reduction techniques are often useful to assist with this type of task. In studies of the overall population of persons with serious mental illness, empirically derived typologies have been used to separate clients into groups based on symptomatology and/or on a mix of client characteristics and demographics (Herman \& Mowbray, 1991). The purpose of a typology is to organize the large amount of information available on individuals in terms of key factors related to treatment and service needs. Cluster analysis is a multivariate statistical procedure which identifies and establishes groups of individuals (clusters) who are similar on a selected sample of attributes (variables) (Blashfield \& Aldenderfer, 1988). The goal is to identify client types that are clearly distinguishable, naturally occurring, and meaningful. If significant descriptors of the homeless mentally ill (HMI) could be agreed upon and clusters derived and validated, then descriptive population studies could report on the percentage from given clusters found, by referral site, from each geographical area - rather than presenting extensive listings of central tendency and variation of vast inventories of variables, which are difficult to interpret. Further studies could then go on to quantify the relevant characteristics of service systems or referral sites that relate to variations in cluster representation.

This report presents descriptive data on a group of HMI individuals, served by a two-site demonstration project, funded by NIMH (Mowbray, Cohen \& Bybee, 1991). The project derived referrals from three different sources: shelters, psychiatric hospital units, and the community mental health agencies' case loads. The study population included those who were literally homeless (undomiciled) as well as the potentially homeless (precariously housed, residentially unstable, and/or about to become undomiciled). Analyses provide overall descriptions of the sample, and examine differences across major client variables (age, gender, race, substance 
abuse) and contextual variables (site, referral source). The results of a cluster analysis are given, including descriptions of cluster members on external, client-descriptor variables and examination of the prevalence of clusters across sites and referral sources. Validation data for the cluster solution are also presented, as are comparisons with a representative sample of seriously mentally ill (SMI) people across the state. Our discussion compares the descriptive results with those from the cluster analysis approach, and makes recommendations for future validation studies.

\section{METHOD}

\section{Sites}

The project was sited in two Michigan communities. Factorytown was characterized by several large manufacturing firms, recent waves of plant closings, and high unemployment rates. Affordable housing was plentiful but often deteriorated or located in high crime or drug use areas. In contrast, Collegetown offered less affordable and more expensive housing, reflecting the impact of two local universities and a proportionately larger white collar/professional population. Both communities had comprehensive, county-based community mental health $(\mathrm{CMH})$ service boards that prioritized services to seriously mentally ill adults. These two CMH Boards operated the demonstration project. The project recruited homeless, mentally ill adults from three types of settings (shelters, psychiatric inpatient units, and active community mental health case loads) in each of the two counties.

\section{Eligibility}

Participants referred from any of the settings were accepted providing that they presented serious mental illness and extreme residential instability, they were county residents, and their next living arrangements had not already been determined. Serious mental illness was defined as a diagnosis of psychotic or major mood disorder or a severe personality disorder coupled with multiple admissions to inpatient or community acute care settings within the last year. Clinical diagnoses determined by psychiatric staff based on hospital or CMH assessment protocols were used to determine psychiatric eligibility for individuals referred from inpatient and $\mathrm{CMH}$ settings. For those recruited from shelter settings, historical diagnosis from $\mathrm{CMH}$ records were used, where available, supplemented by observational ratings developed in earlier research and found to produce reliable discrimination 
of mentally ill individuals when used by shelter staff trained on videotaped vignettes (Cohen, 1988). Diagnoses for individuals recruited from shelter settings were later determined through formal $\mathrm{CMH}$ intake assessment.

\section{Sample}

The current research involved 108 individuals who accepted some project help between October 1, 1989 and December 31, 1990 and whose functioning was assessed by project staff. During this time frame, 55 other individuals accepted project help but were not assessed. In 36 cases, functioning assessments were not available because individuals accepted only limited help from the project, declining to complete a full intake interview or to participate in developing an intervention plan. For the remaining 19 cases, missing assessment protocols were disproportionately common in the Factorytown site ( 27 vs. $4 \%$ at the Collegetown site) and in the shelter $(40 \%)$ and hospital $(20 \%)$ vs. CMH recruitment settings (5\% missing). Missing functioning data were not disproportionate by any other variable, including diagnostic group, age group, sex, race, or substance-abuse problem.

Of the 108 assessed participants, $52.8 \%$ were from Factorytown and $47.2 \%$ were from Collegetown. Over half were recruited from CMH case loads $(53.7 \%$ ), with $29.6 \%$ from psychiatric inpatient units and $16.7 \%$ recruited from shelters. (It should be noted that individuals found at the shelter who were on the $\mathrm{CMH}$ case load were classified into the $\mathrm{CMH}$ recruitment category.) The study participants were relatively young (median age $=35.16$ years, range 20 to 71 ), majority male $(62 \%)$ and white $(61.7 \%)$, with a substantial number $(29.6 \%)$ identified at screening as having substance-abuse problems. The most common diagnosis was schizophrenia $(66.0 \%)$, followed by major mood disorders (18.9\%).

Nearly half $(49 \%)$ had spent some portion of the prior year in a homeless-type setting (shelter, street, temporary room), staying a median of 10 days, and nearly half $(45.4 \%)$ had one or more psychiatric hospitalizations during the baseline year, with a median total stay of 21 days. Only $8 \%$ had any baseline time in jail or prison. Fewer than one third $(32.0 \%)$ had spent any time in a community-supervised dependent setting (group home, adult foster care). However, $64 \%$ had spent more than 6 months in an independent community setting, $32 \%$ had lived with relatives, and $32 \%$ had lived with friends or significant-others during some part of the baseline year. They were relatively nontransient, with only $39 \%$ having had any noninstitutional days outside the local county in the previous year. At project intake, $40.8 \%$ were in homeless-type settings; $13.6 \%$ were in inpatient settings 
with no permanent residence to return to after discharge; $39.8 \%$ were in independent-type settings but were facing imminent eviction or other housing crisis (more than one third of these were with relatives); and 4.8\% were in supervised dependent settings (e.g., adult foster care, group home) in which they could not or refused to remain.

\section{Data Collection}

Intake data were gathered through four methods: referral forms, client interviews, ratings by outreach workers, and examination of archival records.

\section{Referral Forms}

Basic demographic information was provided at the time of screening and referral through use of a standardized screening instrument. A detailed chronology of residential history for the prior 12 months was also provided through this instrument, with information gathered by agency staff from a variety of sources - records, relatives, and clients themselves. To alleviate pressures on hospital staff, project research staff assisted in the process of gathering intake information for individuals in inpatient settings.

\section{Client Interviews}

Client intake interviews were conducted by project outreach workers, trained by the project director. The interview included the Brief Symptom Inventory, a self-report symptom inventory with acceptable reported internal consistency ( $\alpha>.70$ for all subscales) and stability (over a 2-week interval, $r=.90$ for the total index), and some evidence of construct and predictive validity (Derogatis \& Melisaratos, 1983). In this sample, the Global Severity Index of the BSI showed internal consistency of $\alpha=.96$.

Intake interviews also included extensive exploration of individuals' housing, services and support experiences, and preferences, using an adapted version of the Consumer Housing and Support Preference measure (Tanzman, 1990). Descriptive and psychometric analyses of these data are reported in Yeich, Bybee, Mowbray, and Cohen (1993). 


\section{Functioning Ratings}

Client functioning at intake was assessed through ratings of outreach workers using the Client Level Assessment Measure (CLAM; Herman \& Mowbray, 1991; Hazel, Herman, \& Mowbray, 1991). This measure was developed in a statewide study of about 2,500 seriously mentally ill clients of the public mental health system in Michigan. The instrument contains three main types of items: independence items assess the degree to which an individual requires assistance to accomplish specific living tasks (e.g., laundry, keeping appointments), with response options ranging from total independence to near total dependence; cognitive/emotional items assess the frequency of specific symptoms (e.g., trouble sleeping, hearing things that are not there), ranging from never to almost always; and maladaptive behavior items reflect the frequency of specific problematic actions (e.g., property damage, suicide attempt), ranging from never to more than once a day). The original measure produced 16 reasonably independent scales with high internal consistencies and acceptable interrater agreement on most items. To facilitate data collection and to enhance the relevance of the measure to housing and community living issues, the instrument was shortened from 92 to 36 items. Six of the original scales were dropped due to limited relevance for individuals living in community settings. From the remaining scales, items with lower corrected item-total correlations were deleted, leaving the defining items of 10 scales in the revised instrument.

Scaling of the shortened measure with the current sample paralleled the original on most dimensions, with the following exceptions: (a) the interpersonal problems scale showed low internal consistency $(\alpha=.44)$ and was dropped; (b) the medical intervention showed unreliability across raters and was deleted; and (c) two items - financial management problems and medication management skills - failed to load with other community living items and, given their perceived importance to independent living, were retained as single items. Internal consistencies of the remaining scales, as well as intercorrelations among them can be found in Table I. Note that, for all scales, lower scores indicate better functioning.

Outreach workers also assessed participants on the Global Assessment Scale (GAS; Endicott, Spitzer, Fleiss, \& Cohen, 1976), which provides a single numerical indicator of the client's functioning from 1 (lowest) to 100 (highest). Scores below 60 on the GAS indicate moderate to severe deficits. 


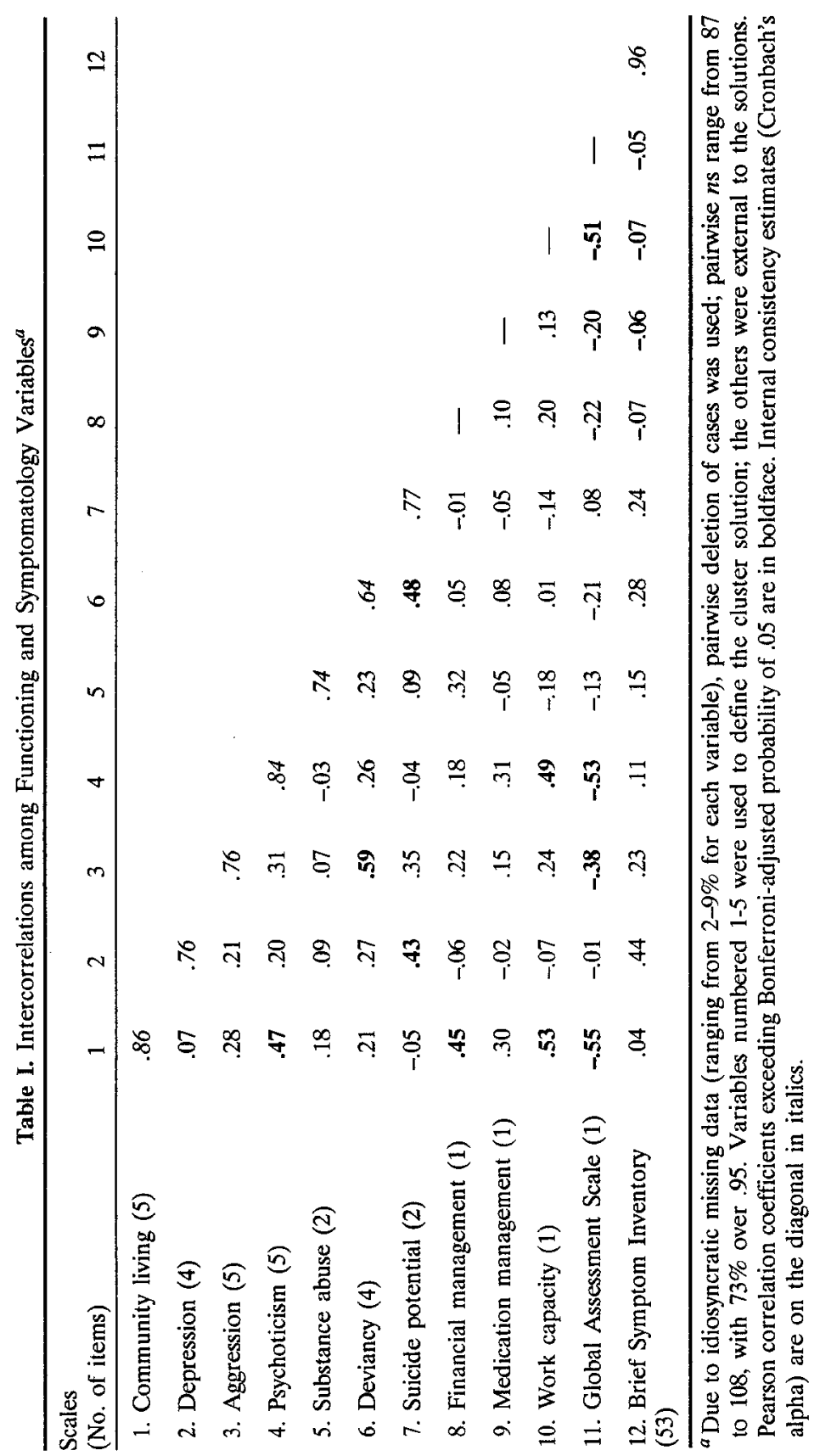




\section{Archival Records}

Archival sources were used for data about baseline psychiatric hospitalization and use of homeless shelters. Trained research assistants (graduate students in the social sciences) searched the records of three inpatient psychiatric units (one state hospital and two community units) and seven homeless shelters that served the project counties.

\section{Data Analysis}

\section{Descriptive Analyses}

Descriptive statistical analyses were carried out on the entire sample. Measures of residential history were extremely positively skewed, with distributions typically characterized by $50 \%$ or more of the sample at 0 and the rest ranging as high as 360 days in a given type of residential setting during the baseline year. Failing to find a transformation capable of correcting the serious skew, we dichotomized these variables into approximately equal groupings. Subgroups on sex, race, age group, presence of substance-abuse, county, and recruitment setting (shelter, hospital, CMH) were examined for differences on residential history, functioning, self-reported symptoms, and diagnosis. For continuous variables (functioning, self-reported symptoms), a multivariate analysis of variance was conducted for each subgroup comparison; if overall group differences were significant, follow-up univariate tests were done, along with Tukey pairwise comparisons, where appropriate. For categorical variables (diagnosis, residential history), chi-square tests were done with Bonferroni-adjusted probability levels reflecting the number of comparisons made; if appropriate, pairwise comparisons were done using a nonparametric analog to the post-hoc Scheffé test (Marascuilo \& McSweeney, 1977).

\section{Cluster Analysis}

Cluster analysis was used to identify groups of individuals whose functioning showed similar levels and patterns. To facilitate comparison with the statewide sample (Herman \& Mowbray, 1991), similar overall clustering strategies were used, adapted to fit the current data set. To ease interpretation and to reduce overfitting to the relatively small sample, a limited set of five functioning assessment scales was used to define the clusters. These five scales - community living problems, depression, 
aggression, psychoticism, and substance-abuse - were chosen because they reflected dimensions thought to be important for community functioning (four were comparable to the six variables used in the statewide study), they showed high internal consistency and reasonable distributions in the current sample, and they appeared to measure relatively independent constructs. Relative independence among input variables is important in cluster analysis to avoid overweighting constructs reflected in more than one measure (Aldenderfer \& Blashfield, 1984). As can be seen in Table I, only 1 of the 10 correlations among the defining variables psychoticism and community living problems - was significant; these concepts seemed sufficiently distinct to warrant the inclusion of both scales.

To adjust for magnitude differences among the response scales, all variables were standardized. Normalized Euclidean distances were used to calculate interindividual similarities, reflecting not only interindividual profile similarities (relative magnitude) but also similarities in absolute magnitude (Blashfield \& Aldenderfer, 1988); thus clusters reflected groupings of individuals similar in level and relative pattern of functioning.

The cluster analysis was done in two stages. Initial groupings were derived by Ward's method, a hierarchical agglomerative technique minimizing within-cluster variation. The centroids of these initial clusters were submitted to an iterative clustering procedure (K-means) to refine final cluster membership and reduce the incidence of cluster misassignment common with agglomerative methods (Blashfield \& Aldenderfer, 1988). To accommodate small amounts of idiosyncratic missing data (ranging from 2 to $9 \%$ for individual variables), pairwise deletion was used for computation of statistics in both stages.

The number of clusters was determined by an inspection of the plot of fusion coefficients and a review of the meaningfulness of the clusters. The most marked flattening of the scree curve occurred at the three-cluster solution. This solution would have resulted in three clusters labeled (from inspection of cluster differences on defining variables) Hostile-aggressive, Depressed, and Best-functioning. However, another sizable decline in slope appeared at the four-cluster point; the four-cluster solution also represented the first large jump in fusion coefficient during the agglomerative process. Inspection of the clusters defined by each solution revealed significant and theoretically meaningful differences between the fourth cluster and the other three. Consequently, the four-cluster solution was chosen, dividing the original Depressed cluster into a Depressed (nonsubstance abusing) and a Substance-abusing cluster and retaining the original Hostile-psychotic and Best-functioning clusters. 


\section{Comparison with Statewide Sample of Seriously Mentally III Individuals}

To enhance the descriptive value of this study, the current sample of homeless mentally ill individuals was compared with a $10 \%$ representative sample of all seriously mentally ill individuals on $\mathrm{CMH}$ case loads in the State of Michigan. The latter data set was collected in 1987, using the original CLAM that was shortened and adapted for this research (Hazel et al., 1991; Herman et al., 1988). Although the scales and clustering procedures were not identical in the two data sets (the scales for the current research being based on items most predictive of the original scale scores), they were similar enough to allow meaningful comparisons. Simple Bonferroni-adjusted $t$-tests were used to test for significant mean differences on functioning scales; chi-square tests (Bonferroniadjusted) were used to assess differences in proportions on diagnoses and cluster membership.

\section{RESULTS}

\section{Results of Descriptive Analyses}

Table II contains descriptive statistics on client functioning and symptomatology variables. Significant multivariate differences were found by county, referral source, and presence of a substance-abuse problem; results of overall MANOVAs, follow-up univariate ANOVAs, and pairwise comparisons are also in Table II. Collegetown clients showed more functioning problems than Factorytown clients (more depression, substance-abuse, deviancy, suicidal potential, medication management problems, and symptomatology), although they were rated as having higher work capability. Clients referred from hospital settings were also less functional (more depression, psychoticism, substance-abuse, deviancy, and symptomatology) than clients recruited from shelter or $\mathrm{CMH}$ settings. Individuals identified at screening as substance-abusers showed more problems on community living, substance abuse, deviancy, financial management, and symptomatology, although they were rated higher in capacity to work. One-way MANOVAs showed no significant differences on functioning and symptomatology variables by sex, race, or age group.

Diagnostic differences were found between subgroups defined by county, sex, and race. Substance-abuse was more prevalent in Collegetown, where $40.4 \%$ were described at referral as having substance-abuse problems (compared with $17.7 \%$ in Factorytown, $\chi^{2}=6.65$, Bonferroni-adjusted 


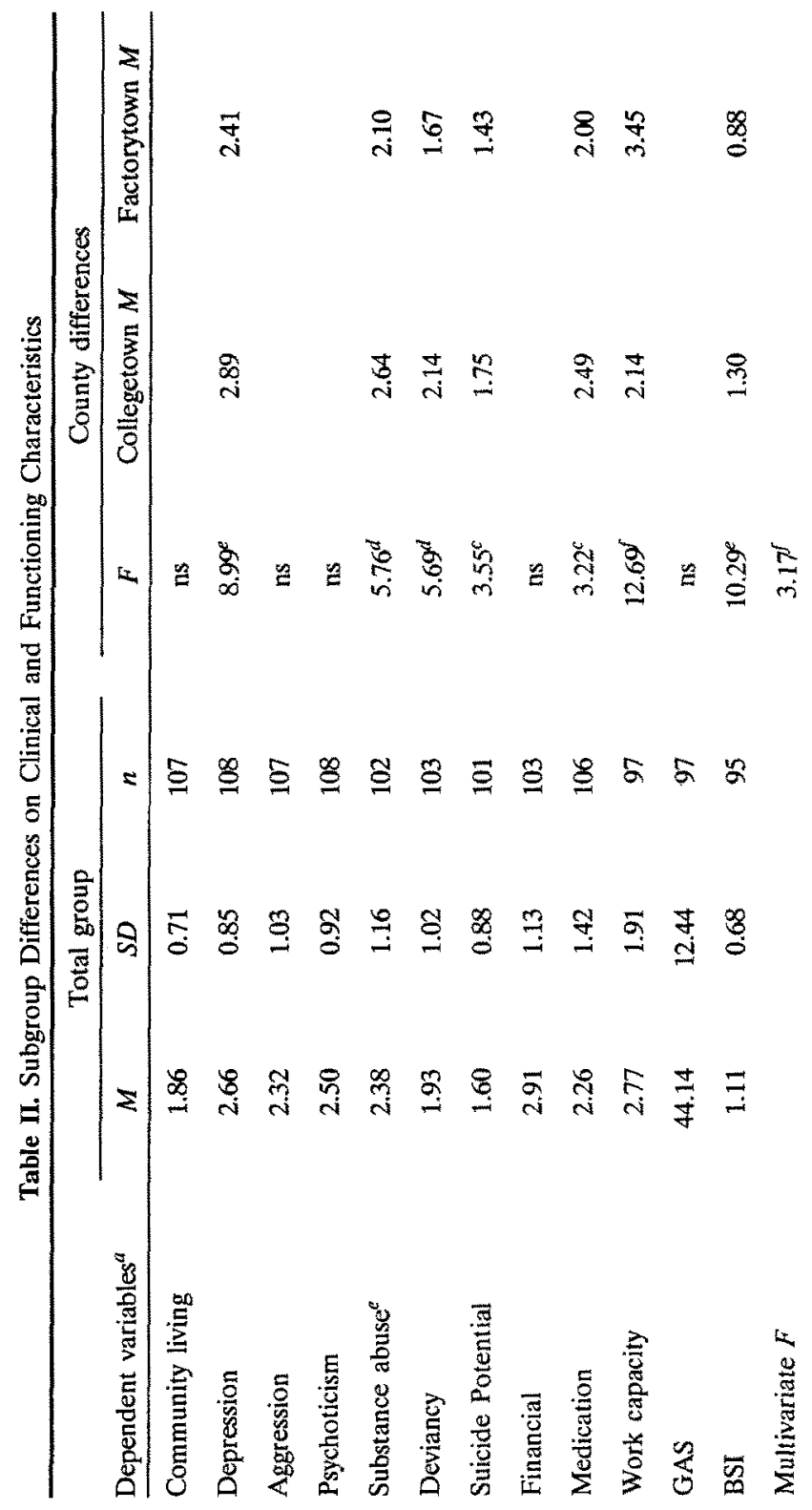




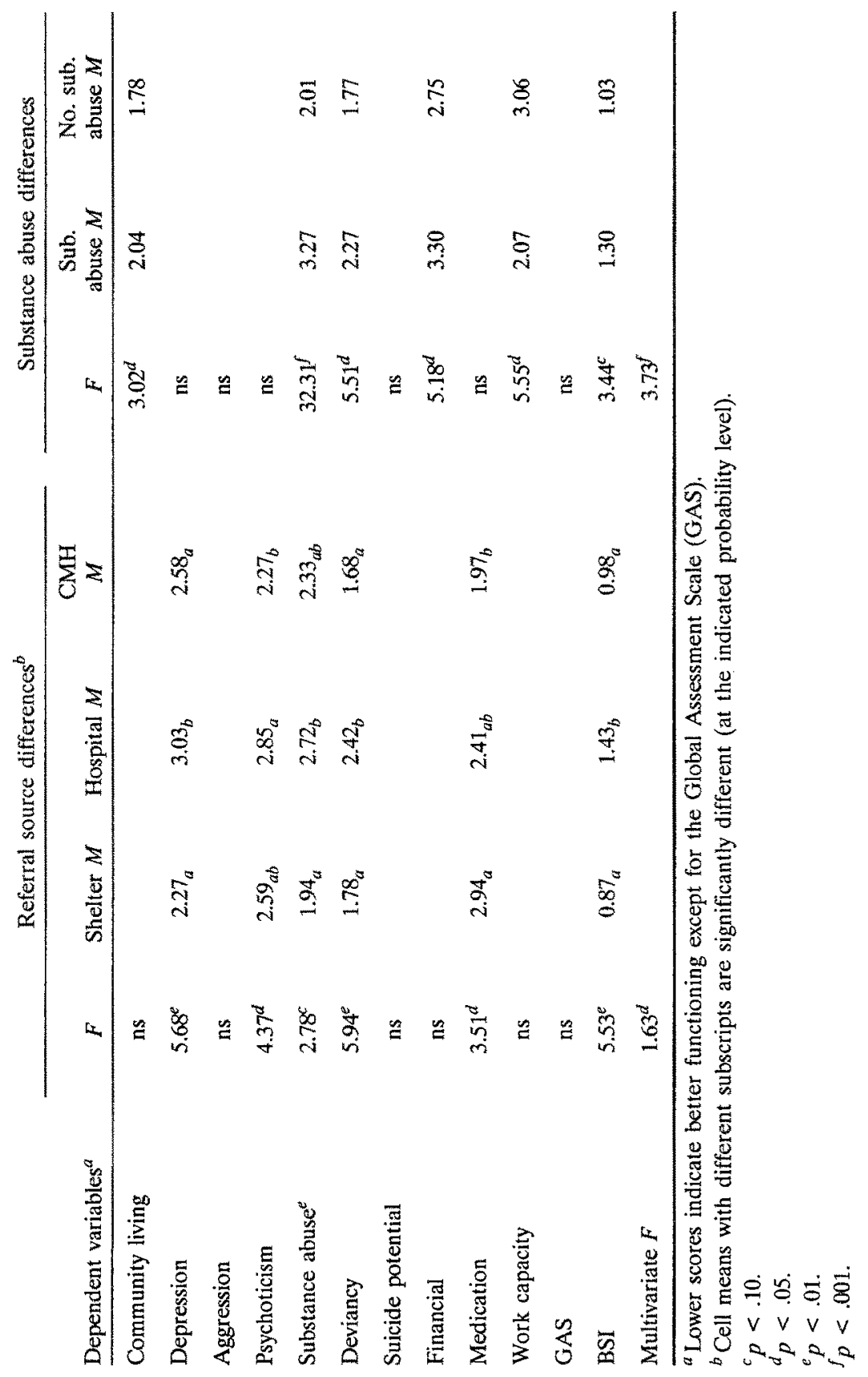


$p<.05$ ) and $21.4 \%$ had a psychiatric diagnosis involving substance-abuse (compared with $6.0 \%$ in Factorytown, $\chi^{2}=5.18$, adjusted $p<.05$ ). Men also had a higher substance-abuse prevalence, with $37.3 \%$ labeled as having substance-abuse problems (vs. $17.1 \%$ for women, $\chi^{2}=5.10$, adjusted $p<.05$ ) and $19.1 \%$ having a substance-abuse diagnosis (vs. $1.8 \%$ for women, $\chi^{2}=9.39$, adjusted $p<.01$ ). Mood disorder diagnoses were more common among whites $(26.6 \%)$ than minority individuals $\left(7.3 \%, \chi^{2}=6.00\right.$, adjusted $p<.05$ ), and borderline personality disorders were more common among women $(17.9 \%)$ than men $\left(1.2 \%, \chi^{2}=10.95\right.$, adjusted $\left.p<.01\right)$. No subgroup differences were found for schizophrenia diagnoses.

Subgroup differences were also found on baseline residential experiences. Baseline stays in homeless-type settings (shelter, street, jail, temporary room) were more common at the Collegetown site (70.4 vs. $28.3 \%$ at Factorytown, $\chi^{2}=17.63$, Bonferroni-adjusted $p<.01$ ), among those referred from shelters $(93.3 \%$ vs. $53.6 \%$ from hospitals and $38.6 \%$ among CMH referrals, $\chi^{2}=14.34$, adjusted $p<.001$ ), among those with substance abuse problems ( 71.0 vs. $42.0 \%$ among nonsubstance abusers, $\chi^{2}=7.17$, adjusted $p<.05$ ), and among men ( 62.3 vs. $33.3 \%$ of women, $\chi^{2}=7.99$, adjusted $p<.05$ ). Baseline stays with relatives were more common in Factorytown (45.7 vs. $20.4 \%$ in Collegetown, $\chi^{2}=7.30$, adjusted $p<.05)$ and among the younger age groups $(51.6 \%$ among those 30 and younger, $36.8 \%$ among those aged 31 to 40 , and $6.5 \%$ among those over $40, \chi^{2}=15.19$, adjusted $p<.01$ ). No subgroup differences were found on number of baseline moves; days outside the local county; days in supervised dependent, independent, or treatment settings; or days in settings with friends or significant others.

\section{Results of Cluster Analysis}

Table III presents the cluster means for variables used to define cluster solution (top section) and the means for functioning and symptomatology variables external to the cluster solution (bottom section). Although standard scale scores were used in the computation of the cluster analysis, raw score means are presented in Table III to facilitate magnitude comparisons. Examination of cluster differences on defining variables led to the assignment of descriptive labels: Cluster 1 (hostile/psychotic) was the largest subgroup at $35.2 \%$ and had the highest mean scores on the aggression and psychoticism scales (as well as the highest score on the community living problems scale); Cluster 2 (depressed) included $18.5 \%$ of the sample and had the highest score on the depression scale coupled with a low score on substance abuse. Cluster 3 (best functioning) was the second largest 


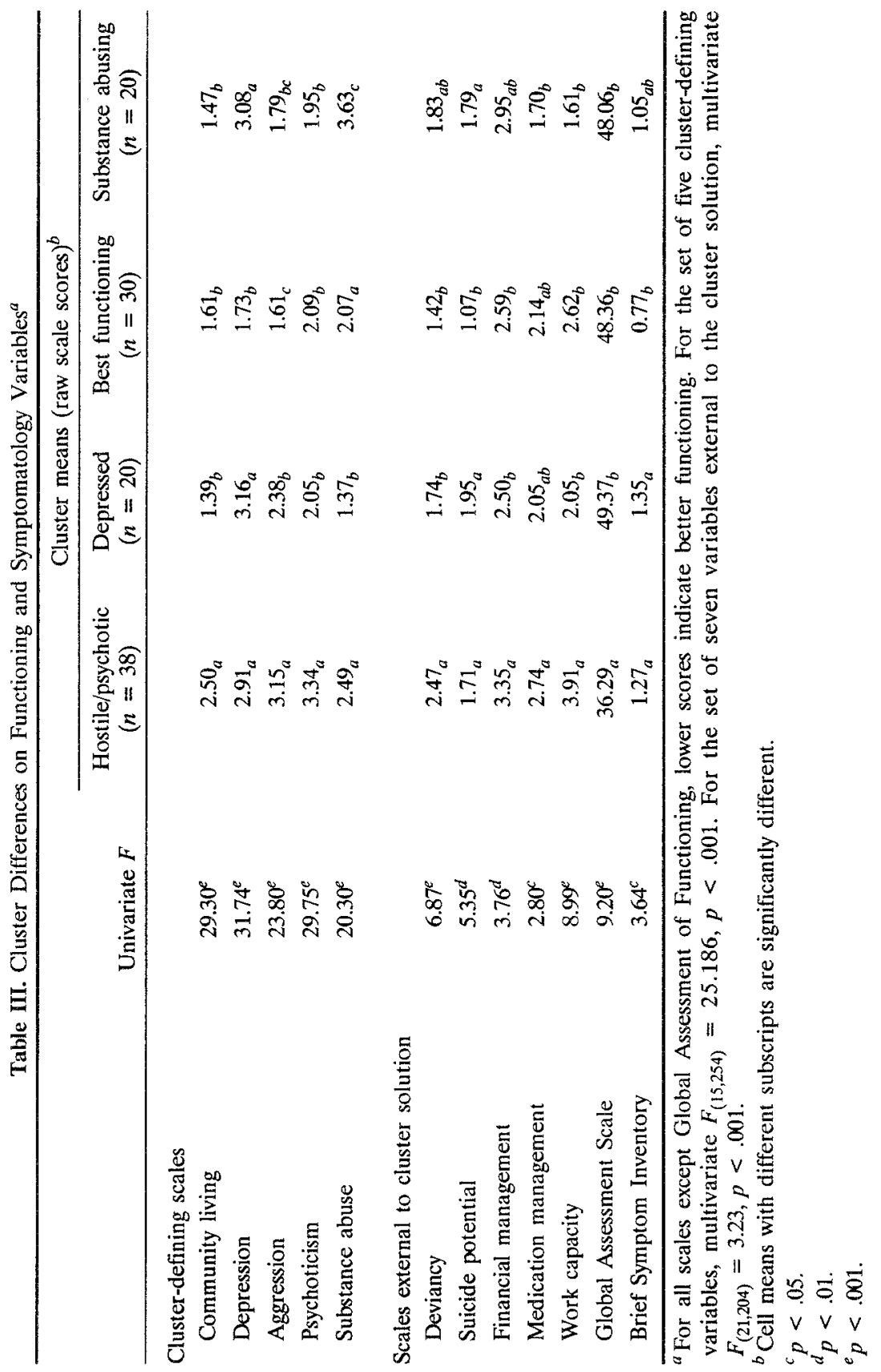


group (27.8\%) and had low scores across all scales, indicating fewer than average problems in all areas. Cluster 4 (substance abusing), a small subgroup $(18.5 \%)$, had by far the highest score on substance-abuse, coupled with the second highest score on depression.

The bottom section of Table III lists cluster means for functioning and symptomatology variables external to the cluster solution. Both the overall MANOVA and all follow-up univariate ANOVAs for these seven variables were significant. The Hostile/psychotic cluster showed the worst functioning on deviancy, financial management problems, and work capacity, as well as the worst GAS. The Best-functioning cluster had significantly lower suicide potential and indicated the least amount of symptomatic distress on the Brief Symptom Inventory. Surprisingly, the Substance-abusing cluster had the lowest (best functioning) mean scores on both medication management and work capacity, although they were significantly better only compared with the Hostile/psychotic cluster. The Depressed cluster showed no significant differences from the Substance-abusing cluster on any of the functioning and symptomatology measures external to the cluster solution.

Table IV presents cluster comparisons on demographic, diagnostic, and other descriptive variables. Significant differences were found on referral source and diagnosis, with nonsignificant trends on age, sex, and baseline psychiatric hospitalization. Most of these differences paralleled the cluster definitions. On age, those 30 and younger were disproportionately common in the Substance-abusing cluster and uncommon in the Bestfunctioning cluster, while those over 40 were disproportionately found in the Hostile/psychotic cluster and absent in the Substance-abusing cluster. There was a marginally significant trend $(p<.07)$ toward women being overrepresented in the Depressed cluster and underrepresented in the Substance-abusing cluster. No significant differences were found on race, county, or substance-abuse problem identified at referral.

Individuals recruited from homeless shelters fell into only two clusters - no clients from this setting were represented in either the Depressed or Substance-abusing clusters. (As previously noted, individuals who had open $\mathrm{CMH}$ cases were categorized as recruited from the $\mathrm{CMH}$ case load even though they might have been initially identified by project staff at a homeless shelter.) Despite this, the clusters did not differ significantly on baseline shelter stays: $30 \%$ of the Depressed cluster and 35\% of the Substanceabusing cluster had, according to archival shelter records, stayed at a shelter at least once during the 1-year baseline period.

As one would expect, members of the Best-functioning cluster were significantly less likely to have been recruited from a psychiatric hospital. Compared with the other three clusters, a smaller proportion of the BestFunctioning cluster had any psychiatric hospitalization during the baseline 


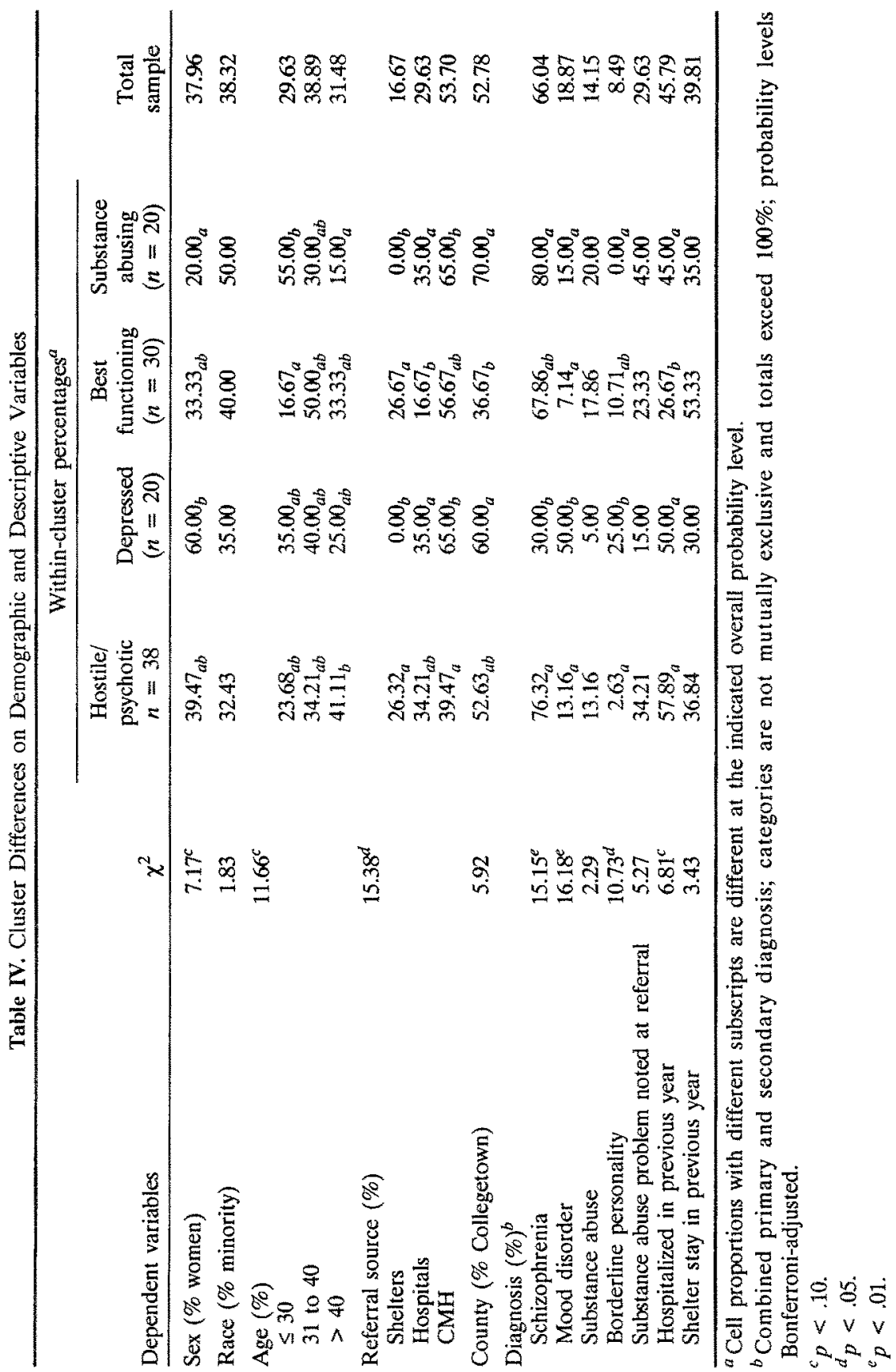


period ( 26 vs. $53 \%$ ); although marginally significant $(p<.06)$, this trend is clearly in the expected direction. Although a substantial proportion (39\%) of the Hostile/psychotic cluster members had been referred from the $\mathrm{CMH}$ case load, they constituted a smaller number than one would expect, given that $54 \%$ of total referrals came from this source. A disproportionately high percentage of both the Depressed and the Substanceabusing clusters were referred from $\mathrm{CMH}$.

Diagnostic differences generally confirmed the functioning variations on which the clusters were derived. Individuals with a diagnosis of schizophrenia made up the largest diagnostic group of all but one cluster, and they were overrepresented in the Hostile/psychotic and Substance-abusing clusters. Schizophrenia was less common in the Depressed cluster, where mood disorders were the most common diagnosis. In the Depressed cluster, borderline personality was also a common diagnosis, in significant contrast to the other clusters. Psychiatric diagnoses involving substance abuse were distributed fairly evenly across all clusters except the Depressed group, where only $5 \%$ were so diagnosed. This apparent difference was not statistically significant. Apparent differences in substance-abuse problems indicated at screening were also not significant, although mean differences on both substance-abuse variables were in the direction one would expect, with higher proportions in the Substance-abusing cluster.

In addition to cluster consistency within the homeless sample, the cluster solution showed strong similarities to the clusters found in the statewide sample of the seriously mentally ill. The statewide study found 6 clusters, 4 of which were replicated in the current study. Cluster composition was similar for these four. Rankings of mean scores across clusters were identical for both samples on all comparable scales except psychoticism and work capacity, which for the Substance-abuse cluster, were (nonsignificantly) ranked second worst in the state sample and best in the homeless sample. Of the two statewide clusters not found in the homeless sample, the Poorest Functioning/High Health Needs cluster was disproportionately found in hospital or dependent care settings and the Suicidal Aggressive cluster was disproportionately identified in rural areas. Failure to find these two clusters in the homeless sample may be partially due to setting dissimilarity.

\section{Results of Comparison with Statewide Sample of Seriously Mentally Ill}

Comparisons of the current sample with the statewide CMH sample of seriously mentally ill individuals are presented in Table V. In terms of diagnostic categories, the homeless sample, compared to the statewide 
community sample, had a similar percentage of schizophrenics and individuals with mood disorders but significantly more diagnosed substanceabusers (12.1 vs. $6 \%$ ) and individuals with borderline personality disorders ( 7.9 vs. $2.6 \%$ ). The homeless group included significantly more individuals from the Hostile/psychotic cluster (35.2 vs. 19.25\%) and significantly fewer from the Depressed (18.5 vs. 25.6\%) and Best-functioning clusters (27.8 vs. $45.4 \%$ ). To explore the possibility that those who could not be included in the cluster analysis (the 55 individuals who accepted limited help and were not assessed) could have altered these comparisons, cluster membership was estimated from referral source, the only variable found to associate with both missing assessment and cluster membership. Using simple proportional extrapolation, an estimated $38.7 \%$ of the entire homeless group $(N=163)$ were in the Hostile/psychotic cluster, $16.6 \%$ in the Depressed, $28.2 \%$ in the Best-functioning, and $16.6 \%$ in the Substanceabusing cluster; use of these estimated percentages would produce no change in the comparison with the statewide sample.

On functioning assessment data, the homeless sample was comparable to the statewide sample on community living problems, depression, medication management, and GAS. Using Bonferroni-adjusted probability levels, 6 of 10 comparisons were statistically significant: on deviancy, psychoticism, aggression, suicidal potential, substance-abuse, and work capacity, the homeless sample showed poorer functioning than the statewide community sample.

\section{DISCUSSION}

\section{Comparisons with Other Homeless Mentally Ill Populations}

As have many other studies, this research contributes to our overall knowledge about individuals who are homeless and mentally ill - their diversity across geographical locations and their similarities. Similar to other studies, our study group was young: $68.3 \%$ were under age 40 , as compared to 68 to $75 \%$ under 40 , reported by Tessler and Dennis (1989) across NIMH-funded demonstration studies of the homeless mentally ill. The racial composition of our homeless group mirrored that of the two county sites, with African Americans somewhat overrepresented. Our population had a somewhat lower percentage of males than reported for the NIMH studies (62\% compared to 68 to $93 \%$ ). This may reflect the fact that referral sites for this project included women's and family shelters, as well as hospitals and CMH. Similar to the conclusions of other studies, our sample was predominantly local area residents $(61.0 \%$ had resided in the county at least a year), although, proportionately fewer than that reported 
Table V. Comparison of Homeless Sample with Statewide Population of Individuals with Serious Mental Illness

\begin{tabular}{llcc}
\hline Dependent variables & $\begin{array}{c}\text { Comparative } \\
\text { statistic }\end{array}$ & $\begin{array}{c}\text { Homeless } \\
\text { sample } \\
(N=108)\end{array}$ & $\begin{array}{c}\text { Statewide } \\
\text { sample } \\
(N>2000)^{a}\end{array}$ \\
\hline Sex (\% women) & $\chi^{2}=6.93^{g}$ & 38.0 & 50.9 \\
Race (\% minority) & $\chi^{2}=6.58^{g}$ & 38.3 & 27.0 \\
Age & $t=-4.91^{g}$ & $M=37.8$ & $M=43.0$ \\
Diagnosis (\%) & & & \\
Schizophrenia & $\chi^{2}=0.03$ & 65.0 & \\
Mood disorder & $\chi^{2}=0.43$ & 22.9 & 64.4 \\
Substance abuse & $\chi^{2}=9.72^{f}$ & 12.1 & 25.0 \\
Borderline personality & $\chi^{2}=15.02^{g}$ & 7.9 & 6.0 \\
Cluster (\%) & & & 2.6 \\
Hostile/psychotic & $\chi^{2}=18.41^{g}$ & & \\
Depressed & & $35.2_{a}$ & $19.3_{b}$ \\
Best-functioning & & $18.5_{a}$ & $25.6_{b}$ \\
Substance-abusing & & $27.8_{a}$ & $45.4_{b}$ \\
\end{tabular}

\begin{tabular}{lccc} 
Functioning scales $^{e}$ & $t$ & $M$ & $M$ \\
\hline Community living & 2.04 & 1.86 & 2.00 \\
Depression & 0.73 & 2.66 & 2.60 \\
Aggression & $5.22^{g}$ & 2.32 & 1.80 \\
Psychoticism & $5.65^{g}$ & 2.50 & 2.00 \\
Substance abuse & $9.40^{g}$ & 2.38 & 1.30 \\
Deviancy & $6.27^{g}$ & 1.93 & 1.30 \\
Suicide potential & $3.42^{f}$ & 1.60 & 1.30 \\
Medication management & 0.44 & 2.30 & 2.32 \\
Work capacity & $3.46^{f}$ & 2.77 & 2.10 \\
Global Assessment Scale & -1.47 & 44.14 & 46.00
\end{tabular}

${ }^{a}$ Due to small proportions of missing data, $N$ for the statewide sample ranged from 2,073 to 2,447 .

${ }^{b}$ Combined primary and secondary diagnosis; categories are not mutually exclusive and totals exceed $100 \%$; probability levels Bonferroni-adjusted.

${ }^{c}$ Two additional clusters in the statewide sample (Poorest Functioning/High Health Needs and Suicidal Aggressive) were not identified in the homeless group; for comparative purposes, cluster percents for the state sample were recomputed, omitting these clusters. Reduced statewide $N$ for this comparison is 1470 .

${ }^{d}$ Cell proportions with different subscripts are significantly different, $p<.05$.

${ }^{e}$ For all functioning scales except the Global Assessment Scale, lower scores indicate better functioning; probability levels Bonferroni-adjusted.

$f_{p}<.05$.

$g_{p}<.01$. 
in other sites (Boston, 76\% and Milwaukee, 68\%; Tessler \& Dennis, 1989). This study's HMI group was similar to other studies' in having few employed ( $4.9 \%$ vs. 3 to $7 \%$ ). The percentage of substance-abusers was also comparable (29.6\% reported at screening compared to a range of 8 to $43 \%$ from the Tessler \& Dennis review).

Because of the detailed residential information we were able to collect, our data can address some of the more general assumptions about the functioning of HMI persons, which have not often been tested in other studies. Thus, we find that $8 \%$ had any time in jail or prison during the baseline year - a percentage undoubtedly higher than the "normal" housed population, but still a small proportion, somewhat in disagreement with Dennis et al.'s (1991) conclusion that HMI individuals have frequent criminal justice involvement. Of our sample $32 \%$ had spent some time living with relatives and the same proportion had lived with friends or significant-others in the baseline year; this seems to contradict conclusions that HMI individuals have few contacts with family or friends (Dennis et al., 1991). Finally, only $18 \%$ of our sample had more than one shelter stay or more than 30 days in a homeless shelter during the baseline year; this does not support assumptions that many of the HMI are chronically homeless.

Thus, as in other research, we find some similarities with HMI population descriptions in the literature (concerning age, residency, employment and substance abuse levels) and some differences (regarding gender, criminal justice involvement, social isolation, and chronic shelter use).

\section{Comparisons with Other Seriously Mentally Ill Populations}

Our research was somewhat unique in also being able to provide a meaningful comparison to a similar population of seriously mentally ill individuals who were not homeless. Often such comparisons are impossible or strained, at best, since populations differ in terms of geographic area and since measurements are quite diverse. In this study, we have the advantage of a previous data set made up of a representative sample of all SMI individuals on CMH case loads in the State of Michigan (Hazel et al., 1991; Herman et al., 1988).

In terms of diagnostic categories, the HMI population, compared to the statewide community sample, had a similar percentage of individuals with schizophrenia and mood disorders, and higher percentages of diagnosed substance-abusers and borderline personality disorder diagnoses. Comparing functioning level data to that from the statewide study, we see that, in many ways, the HMI population was relatively high functioning: the mean GAS 
score was comparable to the larger group's, as were scores for community living problems, depression, and medication management. (It is unlikely that these data characterizing the homeless mentally ill reflect population differences for Factorytown and Collegetown sites, since, in the statewide study, their populations were at or below population means in terms of functioning; e.g., GAS for Factorytown clients averaged 42.68 and 46.27 for Collegetown, compared to 46.00 for the statewide sample.) According to the anchor points closest to our HMI sample's means, this population needs just occasional help or reminders in community living skills, rarely/sometimes has depressive symptoms, and needs occasional reminders for psychiatric medication compliance. However, in the financial management area, the HMI sample needs frequent advice and assistance. This relative functionality seems to contradict existing assumptions about serious deficits in all areas, particularly medication and treatment noncompliance, being characteristic of individuals who are homeless and mentally ill (Dennis et al., 1991). However, in other domains, the HMI clients appear to have more serious deficits than the statewide sample of SMI clients in CMH treatment. That is, their average scores were significantly worse on deviancy, psychoticism, aggression, suicide potential, substance abuse, and work capacity. Like most other SMI groups, the HMI had serious deficits in work skills, with a likely capability of only supported employment or sheltered work. However, the anchors closest to the mean scores on most of these measures do not reflect extreme deficits in other domains. The homeless clients showed deviant behavior and aggression, on average, once a year or less, rarely/sometimes had psychotic symptoms, showed suicidal behaviors once a year or less, and had substanceabuse problems that rarely/sometimes interfered with their functioning. However, as the cluster analysis revealed, these average scores can mask the heterogeneity of functioning among the homeless mentally ill and the extreme dysfunction of a subgroup of clients.

\section{Validation of Cluster Analysis Results}

Examination of cluster differences on functioning and symptomatology measures external to the cluster solution provided some evidence of cluster validity. First, as reported, significant differences were found on all indicators of functioning in the predicted directions. That is, scores were generally very high for the Hostile/psychotic cluster, verifying greatest dysfunction among this group. Similarly, scores were generally low for the Best-functioning cluster. The mean scores for suicide potential and for selfreported symptomatic distress (Brief Symptom Inventory) were highest for the Depressed cluster. The best mean scores for both medication management 
and work capacity appeared for the Substance-abusing cluster, a puzzling finding until one remembers that this cluster was defined by the empirical grouping with community living, aggression, and psychoticism scores indistinguishable from the Best-functioning cluster. The Substance-abusing cluster showed positive functioning skills in all areas except depression and substance abuse.

Some cluster-validating results were also found on demographic and residential variables. For example, the Best-functioning cluster members were least likely to have been recruited from the hospital setting, and the Hostile/psychotic were least likely to come from the CMH case load. Diagnostic categorization data also provided some validation of the cluster solution: in the Depressed cluster, schizophrenia diagnoses were underrepresented, while mood disorders and borderline personality diagnoses were overrepresented. Although not significant, the highest proportion of substance-abuse diagnoses and identified substance-abuse problems occurred in the Substance-abusing cluster. This lack of significance may not be surprising, however, given the oft-noted tendencies of mental health professionals to underidentify substance use (Millman, 1989). Future studies should include additional measures of alcohol and drug use and abuse.

Comparison of these results with the cluster analysis of the statewide seriously mentally ill sample (Herman \& Mowbray, 1991) also provides validation support. In that study, six cluster groups were found: the two groups that are noncomparable to the present results were a Poor Functioning/High Health Needs group, with average age of 56.6 and disproportionate representation of hospitalized individuals (nearly half); and a Suicidal/ Aggressive cluster, a group of younger clients, found primarily in rural areas. It would not be expected that these clusters would emerge in this study, since our sample included only individuals in community settings (or soon to be discharged from inpatient settings) and was drawn only from urban areas. Although the remaining four statewide clusters appeared comparable to the clusters identified in the homeless sample, there were significant distributional differences between the two samples: the homeless sample included more Hostile/psychotic individuals and fewer individuals in the Depressed and Best-functioning clusters.

\section{CONCLUSIONS}

Two different sets of conclusions appear warranted from the present report. First, individuals who are homeless and mentally ill are a diverse, heterogeneous group and, overall, perhaps more like than unlike seriously mentally ill (SMI) persons who are not homeless. Except for one subgroup 
(from the cluster analysis), most individuals exhibit adequate functioning levels and skills. Most problematic is the area of substance abuse, which, along with serious depression, seems to be a major impediment to functioning for one subgroup; substance abuse is a common problem in two other clusters. Another subgroup has major problems with depression (absent substance abuse) and a high suicide potential. However, still another subgroup is functioning well in many areas, although having a documented history of shelter usage. Thus, it may be that individuals who are mentally ill and homeless, overall, are not a qualitatively different group compared to other SMI persons. Rather, many of their differences may result from circumstances and life contexts in which they find themselves. The heterogeneity of the HMI was recently affirmed in a major literature review by Dennis et al. (1991), citing their diversity and the fact that "Not everyone will need one-to-one outreach, and the period of time over which engagement takes place will vary" (p. 1133).

The Hostile/psychotic cluster did manifest serious deficits in many areas and may fit the stereotype of the "chronically mentally ill homeless" which often guides media images and perhaps our expectations for service programs (Bachrach, 1992; Koegel, 1992). Indeed, many of the innovative programs developed for the homeless mentally ill seem designed for this group alone. Thus, there is an emphasis on supported, permanent housing in segregated settings (Lipton, Nutt, \& Sabatini, 1988) and on transitional residences providing intensive rehabilitation to prepare individuals for placement in permanent supportive housing. While structured programs like these may be well-suited for the Hostile/psychotic-type client and the Substance-abusing group as well, more short-term, less intensive interventions seem indicated for the Depressed and Best-functioning groups (which make up nearly half of this study's sample). Structured residential settings for these types of clients are inefficient and may also be detrimental to client outcome if they are incongruent with their living preferences (Plapinger, 1988). Although existing community mental health programs undoubtedly require more adequate resource levels and more innovative programs to deal with all homeless persons, they should be careful about overcommitting to "brick-and-mortar" solutions. We suggest that cluster analysis techniques, as used in the present study, can provide helpful information for future service planning, facilitating identification of subgroups whose characteristics and needs for services may differ.

This point relates to our second major conclusion: Cluster analysis can be a useful tool to better interpret the diversities which homeless mentally ill individuals present. The descriptive results and subgroup analyses that we produced originally were extensive and diffuse. On the other hand, the descriptions of clusters permitted a large amount of individual functioning 
level data to be organized into profiles of clearly distinguishable groups, which was much easier to interpret. Analyzing the distribution of the cluster types, we found clear differences across recruitment sources. Shelters were sites for referral of the Hostile/psychotic and Best-functioning types. The Depressed and Substance-abusing types were already in the service systems - either in hospitals or on the CMH case load (the latter, of course, does not imply they were receiving adequate services). Thus, functioning and clinical characteristics (as reflected in the cluster typology) related most strongly to recruitment site. Except for a marginal trend on hospital days, the clusters did not differ on residential history data. Where clients had been living in the past year may have reflected more about the living arrangements available in their county, or about their personal economic levels or support systems, than it did their current functioning. It also may be that functioning had changed substantially over the previous year, limiting the predictive utility of residential history information.

While the cluster analysis approach appears to have promise for understanding differences among homeless mentally ill individuals, obviously it needs more validation from static and dynamic perspectives. There has been substantial discussion in the literature concerning the need to utilize a common set of dimensions in describing this population (see Fischer \& Breakey, 1991, for example). We hope this report encourages other investigators to do that, as well as to employ cluster analysis methods to validate the present typology. Application of the technique to a larger sample of the homeless mentally ill would be especially useful, as it would allow cross-validation of the cluster solution on random subsamples. Interpretation and validation of cluster analysis results could be enhanced if additional measures were added for some variables (e.g., substance use and abuse, social isolation/social support).

We also encourage other studies to report fully on the characteristics of their recruitment sources. The present study found that recruitment from the shelter, hospital, or CMH case load differed by cluster, but county site did not. It may be that geographical differences found in other studies can be explained more by the prevalence of various recruitment sources (e.g., how many shelters exist and of what type) than by other variations from one location to another. Finally, future analysis should follow members of cluster groups to determine their course of outcome. For example, are the Best-functioning clients more likely to acquire stable housing and get out of the service system? Or do they become worse and move to one of the other clusters? Do the Aggressive/psychotic cluster clients improve with help, or do they deteriorate into jail or psychiatric hospital environments on a long-term basis? 
In summary, descriptive data from this study as well as cluster analysis results demonstrate the heterogeneity of functioning and other characteristics among the homeless mentally ill. The cluster analysis portrays this more clearly, and also shows us that three of the four cluster types have relatively well-developed functioning in most areas. For particular subgroups, interventions may need to focus on depression, substance abuse, or merely income and housing stability (for the Best-functioning group). A subgroup of Hostile/psychotic individuals has major problems in symptomatology, acceptable behaviors, and functioning. This group, stereotypical of the chronically mentally ill homeless, creates major challenges for service providers and requires comprehensive and probably long-term, specialized service interventions. Future challenges involve implementing these individualized interventions and following the cluster membership of homeless mentally ill individuals over time.

\section{REFERENCES}

Aldenderfer, M. S., \& Blashfield, R. K. (1984). Cluster analysis. Beverly Hills, CA: Sage.

Bachrach, L. L. (1992). What we know about homelessness among mentally ill persons: An analytical review and commentary. Hospital and Community Psychiatry, 43, 453-464.

Blashfield, R. K., \& Aldenderfer, M. S. (1988). The methods and problems of cluster analysis. In J. R. Nesselroade \& R. B. Cattell (Eds.), Handbook of multivariate experimental psychology (2nd ed., pp. 447-474). New York: Plenum Press.

Cohen, E. (1988). Consulting to shelters for the homeless: A new role for psychologists. Paper presented at the meeting of the American Psychological Association, Atlanta, GA.

Dennis, D. L., Buckner, J. C., Lipton, F. R., \& Levine, I. S. (1991). A decade of research and services for homeless mentally ill persons: Where do we stand? American Psychologist, 46, 1129-1138.

Derogatis, L., \& Melisaratos, N. (1983). The Brief Symptom Inventory: An introductory report. Psychological Medicine, 13, 595-605.

Endicott, J., Spitzer, R., Fleiss, J., and Cohen, J. (1976). The Global Assessment Scale: A procedure for measuring overall severity of psychiatric disturbance. Archives of General Psychiatry, 33, 766-771.

Fischer, P. J. (1989). Estimating the prevalence of alcohol, drug and mental health problems in the contemporary homeless population: A review of the literature. Contemporary Drug Problems, 16, 333-390.

Fischer, P. J., \& Breakey, W. R. (1991). The epidemiology of alcohol, drug, and mental disorders among homeless persons. American Psychologist, 46, 1115-1128.

Hazel, K., Herman, S. E., \& Mowbray, C. T. (1991). Characteristics of adults with serious mental illness in a public mental health system. Hospital and Community Psychiatry, 42, 518-525.

Herman, S. E., Amdur, R., Hazel, H., Cohen, S., Blondin, P., \& Mowbray, C. T. (1988). Clients with serious mental illness: Characteristics and typology. Lansing: Michigan Department of Mental Health.

Herman, S. E., \& Mowbray, C. T. (1991). Client typology based on functioning level assessments: Utility for service planning and monitoring. Journal of Mental Health Administration, 18, 101-115.

Koegel, P. (1992). Through a different lens: An anthropological perspective on the homeless mentally ill. Culture, Medicine and Psychiatry, 16, 1-22. 
Lipton, F., Nutt, S., \& Sabatini, A. (1988). Housing the homeless mentally ill: A longitudinal study of a treatment approach. Hospital and Community Psychiatry, 39, 40-45.

Marascuilo, L. A., \& McSweeney, M. (1977). Nonparametric and distribution-free methods for the social sciences. Monterey, CA: Brooks/Cole.

Millman, R. B. (1989). Who will treat alcohol and drug abuse patients? Hospital and Community Psychiatry, 40, 989.

Mowbray, C. T., Cohen, E., \& Bybee, D. (1991). Services to individuals who are homeless and mentally ill: Implementation evaluation. New Directions for Program Evaluation, No. 52.

Plapinger, J. (1988). Program services goals: Service needs, service feasibility, and obstacles to providing services to the mentally ill homeless. New York: New York State Psychiatric Institute.

Sosin, M., Piliavin, I., \& Westerfelt, H. (1990). Toward a longitudinal analysis of homelessness. Journal of Social Issues, 46, 157-174.

Tanzman, B. (1990). Researching the preferences of people with psychiatric disabilities for housing and supports: $A$ practical guide. Burlington: University of Vermont, Center for Community Change through Housing and Support.

Tessler, R. C., \& Dennis, D. L. (1989). A synthesis of NIMH-funded research concerning persons who are homeless and mentally ill. Rockville, MD: National Institute of Mental Health.

Yeich, S., Bybee, D., Mowbray, C. T., \& Cohen, E. (1993). Exploring dimensions of consumer housing and support preferences. Manuscript submitted for publication. 